\title{
Asymptotic freedom of Yang-Mills theory with gravity
}

\author{
Sarah Folkerts* ${ }^{*}$ Daniel F. Litim, ${ }^{2}$ and Jan M. Pawlowski ${ }^{1,3}$ \\ ${ }^{1}$ Institut f. Theoretische Physik, Universität Heidelberg, \\ Philosophenweg 16, 69120 Heidelberg, Germany \\ ${ }^{2}$ Department of Physics 85 Astronomy, University of Sussex, Brighton, BN1 9QH, U.K. \\ ${ }^{3}$ ExtreMe Matter Inst. EMMI, GSI, Planckstr. 1, 64291 Darmstadt, Germany
}

\begin{abstract}
We study the behaviour of Yang-Mills theory under the inclusion of gravity. In the weakgravity limit, the running gauge coupling receives no contribution from the gravitational sector, if all symmetries are preserved. This holds true with and without cosmological constant. We also show that asymptotic freedom persists in general field-theory-based gravity scenarios including gravitational shielding as well as asymptotically safe gravity.
\end{abstract}

PACS numbers: 11.10.Hi, 04.60.-m, 11.15.Tk

\section{INTRODUCTION}

Asymptotic freedom of Yang-Mills theories [1, 2] - the weakening of the strong force at asymptotically short distances - is a central characteristics of the Standard Model. Within a renormalisation group language, asymptotic freedom is signaled by a trivial ultraviolet fixed point with vanishing Yang-Mills coupling. This pattern is at the root of the existence of YangMills theory as an asymptotic perturbative series valid for high energies, i.e. energies much larger than the dynamical strong-coupling scale, $\Lambda_{\mathrm{QCD}}$, and makes the high energy behaviour accessible with weak coupling methods. It is well-known, however, that this feature can be destabilized once the couplings to matter degrees of freedom are taken into account, leading to constraints for the latter to ensure asymptotic freedom within the SM and its various extensions.

Less is known about the stability of asymptotic freedom under the inclusion of gravity. Recently, quantum gravitational corrections to the Yang-Mills coupling have been studied perturbatively to one-loop order in Newton's coupling [3 -8], by treating gravity as an effective theory amended by an ultraviolet cutoff of the order of the Planck scale [9] (see [10 12] for earlier work). The strength of this technique is that quantum gravitational fluctuations can be computed in the low energy regime without the knowledge of the underlying quantum theory for gravity. Asymptotic freedom remains intact in these studies and extensions thereof including compact extra dimensions [13, 14] and a cosmological constant [15]. These one-loop results have recently been confirmed beyond perturbation theory in [16].

The findings open up a number of farther reaching questions. The one-loop graviton contribution to the Yang-Mills $\beta$-function comes out regularisation- and gauge-fixing dependent, which raises the question about its universality. It has also been argued that the one-loop coefficient is not invariant under re-parameterisations in field space, and hence not a measurable quantity [17]. For specific gauges or regularisations a vanishing one-loop coefficient has

\footnotetext{
* Present address: Arnold Sommerfeld Center, Ludwigs-Maximilians-Universität, Theresienstr. 37, 80333 München, Germany
} 
been found, and a computation beyond the one-loop level becomes mandatory. Also, the applicability of the effective theory is confined to the regime of weak gravity and strong coupling effects will become important and should be taken into account for energies approaching the Planck scale. Finally, at Planck-scale energies and beyond, an effective theory description is superseded by a fundamental theory for gravity, and the ultimate fate of asymptotic freedom for Yang-Mills theory then depends on the ultraviolet completion for gravity.

In this Letter, we study Yang-Mills theory coupled to gravity with the help of the functional renormalisation group [18 21] (see [16] for a related study). This technique is based on the infinitesimal integrating-out of momentum modes from a path integral representation of quantum field theory by means of an infrared momentum cutoff. The renormalisation group flow links the fundamental theory with the corresponding quantum effective action at low energies. A particular strength of this method is its flexibility, allowing for perturbative and non-perturbative approximations. In the past, it has been applied to Yang-Mills theory [19 31] and gravity [34 44] individually, both at weak and strong coupling, which makes it an ideal tool for the study of the coupled system [45].

In the weak gravity limit, we analyse the gravitational corrections to Yang Mills theory for general regularisations and backgrounds. Gauge and diffeomorphism invariance of the effective action is achieved using the background field method. We evaluate the background field independence of our results by extending earlier explicit results within gauge Yang Mills theory [22] to quantum gravity. In this general setting the gravitational contribution to the running gauge coupling is computed at one-loop. The result encompasses all previous studies, also including a cosmological constant. We also address asymptotic freedom in the limit where the graviton anomalous dimension can become large. Here, we are particularily interested in the asymptotic safety scenario for gravity [33 44, 46]. We also evaluate asymptotic freedom in the scenario where quantum gravity is shielded by black hole formation [47 51].

The outline of this Letter is as follows. We introduce our renormalisation group set-up (Sec. II), and discuss gravitational corrections to Yang-Mills theory within a background field approach (Sec. III). A detailed discussion of the background field dependence is given (Sec. IV), amended by a complementary RG study for flat backgrounds (Sec. V). Our results are compared with earlier findings in perturbation theory at the one-loop order (Sec. VI). Beyond one-loop, we study the leading gravitational corrections to Yang-Mills in the presence of a gravitational fixed point (Sec. VII) and in the presence of a cosmological constant term (Sec. VIII). We close with a brief discussion (Sec. IX).

\section{RENORMALISATION GROUP}

The Functional Renormalisation Group is based on a momentum cutoff for the propagating degrees of freedom and describes the change of the scale-dependent effective action $\Gamma_{k}$ under an infinitesimal change of the cutoff scale $k$ [18 21]. Thereby it interpolates between a microscopic action in the ultraviolet and the full quantum effective action in the infrared, where the cutoff is removed. In its modern formulation, the renormalisation group flow of $\Gamma_{k}$ with the logarithmic scale parameter $t=\ln k$ is given by Wetterich's flow equation [18]

$$
\partial_{t} \Gamma_{k}=\frac{1}{2} \operatorname{Tr} \frac{1}{\Gamma_{k}^{(2)}+R_{k}} \partial_{t} R_{k} .
$$


The trace stands for a sum over indices and a loop integration, and $R_{k}$ (not to be confused with the Ricci scalar $R\left(g_{\mu \nu}\right)$ ) is an appropriately defined momentum cutoff at the momentum scale $q^{2} \approx k^{2}$. The effective action depends on the metric field $g$, the gauge field $A_{\mu}^{a}$ including possible Abelian factors, and the related ghosts $\eta, \bar{\eta}$ and $C, \bar{C}$ for metric field and gauge field respectively. In short we have $\Gamma_{k}=\Gamma_{k}[\phi, \bar{\phi}]$, where the fields are put into a superfield $\phi=(g, \eta, \bar{\eta}, A, C, \bar{C})$. The action may also depend on specifically chosen, non-propagating background fields $\bar{\phi}$. In quantum gravity, the introduction of a background metric is necessary as one has to fix a gauge, or more generally, one has to choose a specific parameterisation of the configuration space. This fact is at the root of the problem of background independence of quantum gravity.

The flow equation (11) is derived from the insertion of infrared cut-off terms in the path integral,

$$
\Delta S_{k}[\phi, \bar{\phi}]=\frac{1}{2} \int d^{d} x \sqrt{\bar{g}}(\phi-\bar{\phi})_{i} R_{k}^{i j}[\bar{\phi}](\phi-\bar{\phi})_{j}
$$

where the regulator $R_{k}$ is a matrix in field space and may also couple different species of fields, in particular metric and gauge fields, and may depend on the background $\bar{\phi}$. Note that the background metric dependence in (2) via $\sqrt{\bar{g}}$ is necessary to keep the one loop nature of the flow equation (11). Furthermore the contraction of the spin one and spin two components in $\phi$ also depend on the background metric.

The rhs. of the flow equation (1) consists of one loop diagrams with full field-dependent propagators multiplied with the scale derivative of the regulator. This entails that for computing a $n$-loop effective action one has to insert the $n$-1-loop effective action on the rhs. If interested in the one loop effective action we therefore have to insert the classical action on the rhs. The structure of the flow equation is such that standard perturbation theory is recovered to all loop orders by iteration [30].

Gauge and diffeomorphism symmetry is encoded in the related Slavnov-Taylor identities [19, 21, 27]. These symmetry identities are modified in the presence of the regulator term (2). In the vanishing cut-off limit, $k \rightarrow 0$, the modifications also vanish and we are left with the standard symmetry identities. Furthermore, there are combined transformations of both, the background $\bar{\phi}$ and the fluctuation $\phi$, which leaves the effective action invariant. It is for that reason that

$$
\Gamma_{k}[\phi]=\Gamma_{k}[\phi, \phi]
$$

is both, gauge and diffeomorphism invariant.

\section{ASYMPTOTIC FREEDOM AND GRAVITATIONAL INTERACTIONS}

In the present work we consider an approximation to the effective action which reproduces the full one loop effective action but allows also to discuss properties beyond one loop. Such an approximation for $\Gamma_{k}[\phi]$ defined in (3) is given by

$$
\Gamma_{k}[\phi, \bar{\phi}]=\int d^{d} x \sqrt{\operatorname{det} g_{\mu \nu}}\left[\frac{Z_{N, k}}{16 \pi G_{N}}\left(-R\left(g_{\mu \nu}\right)+2 \bar{\Lambda}_{k}\right)+\frac{Z_{A, k}}{4 g_{S}^{2}} F_{\mu \nu}^{a} F_{a}^{\mu \nu}\right]+S_{\mathrm{gh}}+S_{\mathrm{gf}}+\Delta \Gamma_{k}
$$


Here we have introduced the Einstein-Hilbert action and the classical Yang-Mills action as well as gauge fixing and ghost contributions $S_{\mathrm{gh}}$ and $S_{\mathrm{gf}}$. The gauge and diffeomorphism invariant action, (3) , is defined as $\Gamma_{k}[\phi]=\Gamma_{k}[\phi, \phi]$ where the gauge fixing term and a part of $\Delta \Gamma_{k}$ vanishes. Due to quantum fluctuations, all couplings have become running couplings and depend on the infrared RG-scale $k$ : the running Newton coupling $G_{N, k}=G_{N} / Z_{N, k}$, the running cosmological constant $\Lambda_{k}$, and the running Yang-Mills coupling $g_{S, k}=g_{S}^{2} / Z_{A, k}$. Further quantum corrections such as higher order curvature invariants and further mattergravity interaction terms can be generated as well and are contained in $\Delta \Gamma_{k}$.

In the present work we discuss the running of the fundamental dimensionless couplings of the theory, the Yang-Mills coupling and the dimensionless Newton coupling,

$$
g_{N}=\frac{k^{2} G_{N}}{Z_{N, k}} .
$$

The running is encoded in the respective $\beta$-functions $\left(\beta_{X} \equiv k \partial_{k} X\right)$ as

$$
\begin{array}{lll}
\beta_{g_{S}}=\frac{1}{2} \eta_{A} g_{S, k} & \text { with } & \eta_{A}=-\frac{\partial_{t} Z_{A, k}}{Z_{A, k}}, \\
\beta_{g_{N}}=\left(2+\eta_{N}\right) g_{N} & \text { with } & \eta_{N}=-\frac{\partial_{t} Z_{N, k}}{Z_{N, k}} .
\end{array}
$$

The above equations (6), (77) reflect the fact that in a background field approach the running couplings run inversely to the wave-function renormalisations $Z$ of the respective fields. The running of the $Z$ 's splits into a pure Yang-Mills part (only Yang-Mills loops/fluctuations) and a gravity part that also contains internal graviton lines,

$$
\eta_{A}=\eta_{A, \mathrm{YM}}+\eta_{A, \mathrm{grav}}, \quad \eta_{N}=\eta_{N, \mathrm{grav}}+\eta_{N, \mathrm{YM}}
$$

In the present work we are specifically interested in the sign $\eta_{A \text {,grav }}$, since $\eta_{A, \mathrm{YM}}$ is proportional to the Yang-Mills coupling and tends to zero in the UV if asymptotic freedom is present. Hence, for $\eta_{A \text {,grav }}<0$ at vanishing YM-coupling, asymptotic freedom is supported by quantum gravity corrections, whereas for $\eta_{A \text {,grav }}>0$ it is spoiled.

So far we have not specified the regulator $R_{k}$. Indeed the full effective action at $R_{k} \equiv 0$ (for $k=0$ ) cannot depend on this choice, however at finite $k \neq 0$ with $R_{k}$ there will be a $k$-dependence. In the following we shall exploit this freedom to disentangle gravity and gauge theory at one loop. To that end we use a specific class of regulators which respects the renormalisation group scalings of the underlying physical theory at $k=0$,

$$
R_{k}[\bar{\phi}]=\Gamma_{k}^{(2)}[\bar{\phi}] r[\bar{\phi}]
$$

The dimensionless regulator functions $r^{i j}$ are demanded to scale trivially under the RGscalings of the underlying theory, and

$$
\Gamma_{k, i j}^{(2)}[\phi, \bar{\phi}]=\frac{\delta^{2} \Gamma_{k}[\phi, \bar{\phi}]}{\delta \phi^{i} \delta \phi^{j}}
$$

are the second derivatives of the effective action w.r.t. the full fields $\phi$. Regulators of the form (9) are called RG-adjusted [21, 28] or spectrally adjusted [23], and scale like (inverse) 
two-point functions. With this property the RG-invariance and scaling properties of the underlying theory carry over to that of the regularised theory [21], and facilitate the study and interpretation of $\beta$-functions w.r.t. to the $t$-scaling. The dimensionless regulator functions $r^{i j}$ depend on covariant Laplacians in the presence of the background $\bar{\phi}$. We specify

$$
\begin{aligned}
r^{g g} & =r^{g g}\left(-\Delta_{\bar{g}} / k^{2}\right) \\
r^{\bar{\eta} \eta} & =-r^{\eta \bar{\eta}}=r^{\bar{\eta} \eta}\left(-\Delta_{\bar{g}} / k^{2}\right) \\
r^{A A} & =r^{A A}\left(-\Delta_{\bar{g}}(A) / k^{2}\right) \\
r^{\bar{C} C} & =-r^{C \bar{C}}=r^{\bar{C} C}\left(-\Delta_{\bar{g}}(A) / k^{2}\right) .
\end{aligned}
$$

All other components vanish. Regulator functions $r$ with (11) are diagonal. They only connect the metric and gauge field with themselves and gosts with anti-ghosts. Note that this is the minimal form of regulator functions that suppress all infrared (or ultraviolet) propagation of all fields. Dropping one of the components in (11) leads to full quantum propagation of the related field. Hence, the above choice naturally disentangles the Yang-Mills and gravitational sectors, and is therefore well-suited for our analysis.

Finally we are interested in the RG-scaling (w.r.t. $k$ ) of the couplings of the background gauge field $\bar{A}$ and the background metric field $\bar{g}$. Inserting the regulator (9) in the flow (1) and setting $\phi=\bar{\phi}$ we arrive at $[2123,28$ 31]

$$
\partial_{t} \Gamma_{k}[\phi]=\frac{1}{2} \operatorname{Tr} \frac{1}{1+r[\phi]} \partial_{t} r[\phi]+\operatorname{Tr} \partial_{t} \Gamma_{k}^{(2)}[\phi, \phi] \frac{1}{\Gamma_{k}^{(2)}[\phi, \phi]} \frac{r[\phi]}{1+r[\phi]},
$$

where $\Gamma_{k}[\phi]=\Gamma_{k}[\phi, \phi]$, see (3). The above RG flow is the key equation in the present work. The first term on the rhs. consists out of all one loop contributions to the flow. The second term has the form of an RG improvement. It consists out of all terms beyond one loop, including all non-perturbative contributions. Note that it vanishes at one loop.

We observe that for the cutoff choice with diagonal $r$ in (11), the one loop flow disentangles. It is the sum of the pure gravity flow driven by $r^{g g}$ and $r^{\bar{\eta} \eta}$, and a pure gauge flow driven by $r^{A A}$ and $r^{\bar{C} C}$. Cross-terms leading to gravity loops for i.e. the gauge field propagators or gauge field loops for the graviton propagators are absent. The full one-loop effective action is then given by

$$
\Gamma[\phi]=\Gamma_{\Lambda}[\phi]+\frac{1}{2} \int_{\Lambda}^{0} \frac{d k}{k} \operatorname{Tr} \frac{1}{1+r[\phi]} \partial_{t} r[\phi],
$$

where $\Gamma[\phi]=\Gamma_{k=0}[\phi]$. It follows from (13) that for the class of cutoff functions (11) the full $\beta$ function of the background gauge coupling, $\beta_{g_{S}}$, in the presence of gravitational fluctuations is given by the standard result without gravity

$$
\beta_{g_{S}}=\beta_{g_{S}, \mathrm{YM}}=-\frac{1}{2} \eta_{A, \mathrm{YM}}
$$

up to corrections starting at the 2-loop level. Beyond one loop, the potential structural disentanglement no longer holds true: $\partial_{t} \Gamma_{k}^{(2)} / \Gamma_{k}^{(2)}$ is not diagonal and hence entangles YangMills and gravity sector. 


\section{BACKGROUND FIELD DEPENDENCE}

The functional renormalisation group approach of Sec. III allows us to classify and understand the scheme dependence of the $\beta$-functions of a general matter-gravity system. In particular this allows us to explain the scheme dependences observed so far for the YangMills - gravity theory, as well as to extract the physics stored in the $\beta$-functions, i.e. the UV stability of the coupled system. We note in this context that the functional RG setup also covers the standard perturbative regularisation schemes such as momentum cutoff, dimensional, Pauli-Villars, or heat kernel regularisation.

Scheme dependences of $\beta$-functions in the background field approach have already been studied for general field theories [21, 22, 28, 29]. In [22] it has been shown that the YMcoupling receives contributions from the background field dependence of the regularisation. These terms even can alter the one-loop $\beta$-functions. It has also been shown explicitly how to extract the standard one-loop $\beta$-function with the help of an equation which tracks the background field dependence of the regularisation. In YM theory, the background field approach is a convenient choice. In gravity, however, it becomes a necessity. There, the background field dependence of the regularisation is encoded in

$$
\int \frac{1}{\sqrt{\bar{g}}} \frac{\delta \sqrt{\bar{g}} R_{k}}{\delta \bar{\phi}} \frac{\delta \Gamma_{k}[\phi, \bar{\phi}]}{\delta R_{k}}=\frac{1}{2} \operatorname{Tr} \frac{1}{\Gamma_{k}^{(2)}[\phi, \bar{\phi}]+R_{k}[\bar{\phi}]} \frac{1}{\sqrt{\bar{g}}} \frac{\delta R_{k}[\bar{\phi}]}{\delta \bar{\phi}} .
$$

It is left to evaluate whether the background field dependence affects the one-loop result (14) for the background coupling as suggested by the above considerations. For the given cutoff functions (9), (11), we derive the regulator-induced background field dependence (15),

$$
\int \frac{1}{\sqrt{g}} \frac{\delta \sqrt{g} R_{k}}{\delta \phi} \frac{\delta \Gamma_{k}[\phi, \phi]}{\delta R_{k}}=\frac{1}{2} \operatorname{Tr} \frac{1}{1+r[\phi]} \frac{\delta r}{\delta \phi}+\operatorname{Tr} \frac{1}{\sqrt{g}} \frac{\delta \sqrt{g} \Gamma_{k}^{(2)}}{\delta \bar{\phi}}[\phi, \phi] \frac{1}{\Gamma_{k}^{(2)}[\phi, \phi]} \frac{r[\phi]}{1+r[\phi]},
$$

already evaluated at $\phi=\bar{\phi}$. Note that the structure of (15) resembles that of the flow equation (12). The first term is purely diagonal and one loop, whereas the second term contains the non-perturbative contributions and entangles gravity and Yang-Mills due to $\left(\delta \Gamma_{k}^{(2)} / \delta \bar{\phi}\right) / \Gamma_{k}^{(2)}$. In contradistinction to the second term in (12), it also contributes at one loop. In particular, contributions proportional to $\delta \Gamma_{k}^{(2)} / \delta \bar{\phi}$ in the gravity sector produce terms

$$
\propto \frac{\delta}{\delta \phi} \int d^{4} x \sqrt{g} \operatorname{tr} F_{\mu \nu} F^{\mu \nu}
$$

if evaluated at $\phi=\bar{\phi}$. Therefore we conclude that in general graviton contributions to the regularisation-induced background dependence of the effective action are created. We have already argued that for the regularisation schemes diagonal in $r$ defined in (11), the graviton-contribution to the $\beta$-function of the background field defined via (12) vanishes. Note that this does not hold for the $\beta$-functions of the fluctuations. However, the $\beta$-function of the background field also contains unphysical contributions from the field-dependence of the regulators. These terms are given by the $t$-derivative of the $F^{2}$-contribution of the right hand side in (16). While the $t$-derivative of the $F^{2}$-contribution of the first term vanishes, this is not so for the one-loop contribution of the second term.

In turn, regularisations which induce a mixing between gravity and Yang-Mills fields are expected to give a non-vanishing contribution already at one-loop for all $\beta$-functions. The 
above arguments entail that the background $\beta$-functions of the coupled YM-gravity system are scheme-dependent even at one-loop. Note, however, that the UV stability of the YMgravity system is solely controlled by the signs of the $\beta$-functions of the fluctuation fields.

In conclusion, the following picture has emerged. UV stability of the YM sector relates to the (negative) sign of the $\beta$-function for the fluctuating gluon. This information can be extracted directly from the correlation functions of the fluctuating gluons. Alternatively, one can exploit the fact that the regularisation-independent part of the $\beta$-function of the background coupling carries the same sign. In general, the second option requires the use of (16).

\section{FLAT BACKGROUNDS}

The results of the last section entail that a computation of the graviton contribution to the Yang-Mills $\beta$-function requires the distinction between fluctating graviton and background graviton. This is possible if the computation is done in a flat background within a standard vertex expansion. Here we put forward a computation of the YM $\beta$-function, which differs from the previous studies in two aspects [45]: Firstly, the analysis is performed for trivial backgrounds $\bar{A}=0$ and $\bar{g}_{\mu \nu}=\eta_{\mu \nu}$. Secondly, we employ classes of momentum cutoffs with a tensorial structure different from (91). The result complements our previous findings and allows to make close contact with all previous 1-loop studies [3, 5, 6, 15] as well as the flow study [16].

This also allows us to make use of the fact that loop contributions of fluctuating gravitons to the correlation functions of the fluctuating gluons and to the background gluons agree: the vertices of the two fields are derived only from the classical YM-action and not from the ghost and gauge fixing terms. The classical YM-action only depends on $\phi=\bar{\phi}+(\phi-\bar{\phi})$ and hence derivatives w.r.t. the background fields $\bar{\phi}$ and w.r.t. the fluctuation fields $(\phi-\bar{\phi})$ agree. In other words, the two possibilities of how to compute the graviton contribution to the $\beta$-function of the fluctuating gluon agree. We emphasise that this does not hold for the gluon contribution to the $\beta$-function of the fluctuating graviton.

Our Ansatz for the effective action is dictated by asymptotic freedom: a good approximation for Yang-Mills theory at high energies is given by the classical $F^{2}$ operator plus a gauge fixing term, both equipped with a running wave function renormalisation $Z_{A, k}$ and a classical scale-independent ghost action. The interaction of the gauge bosons with gravity is induced by the metric appearing in the spacetime integral measure which is now a quantum field. Also adding the Einstein-Hilbert action, we arrive at

$$
\begin{aligned}
\Gamma_{k}[g, A ; \bar{g}, \bar{A}] & =\frac{Z_{N}}{16 \pi G_{N}} \int d^{d} x \sqrt{g}\left(-R+2 \bar{\lambda}_{k}\right)+\frac{Z_{A}}{4} \int d^{d} x \sqrt{g} g^{\mu \rho} g^{\nu \sigma} F_{\mu \nu}^{a} F_{\rho \sigma}^{a} \\
& +\frac{Z_{N}}{2 \alpha} \int d^{d} x \sqrt{\bar{g}} \bar{g}^{\mu \nu} L_{\mu} L_{\nu}+\frac{Z_{A}}{2 \xi} \int d^{d} x \sqrt{\bar{g}}\left(\bar{g}^{\mu \nu} \bar{D}_{\mu}^{a b}(A-\bar{A})_{\nu}^{b}\right)^{2}+S_{\mathrm{gh}}
\end{aligned}
$$

Note that the $k$-dependence of $Z_{N}$ and $Z_{A}$ is not indicated explicitly. In (18), we have a general linear gauge condition $L_{\mu}$ with gauge parameter $\alpha$ for the graviton, and a background 
field gauge with parameter $\xi$ for the gluon. In general, both gauge fixing parameters are $k$ dependent, except for vanishing values. The latter constitutes a fixed point of the flow, [26]. $S_{\text {gh }}$ consists of the sum of the classical ghost action for gravity and the one for Yang-Mills theory. $S_{\text {gh }}$ neither contributes to the graviton-induced corrections to the running of the gluon coupling, nor to the gluon-induced running of the gravitational coupling. There is a simple reason for this. The gravitational ghost term, by construction, does not contain any gauge fields. The Yang-Mills ghost term only couples to the background metric field, eg. $S_{\mathrm{YM}, \mathrm{gh}}=$ $\int d^{d} x \sqrt{\bar{g}} \bar{g}^{\mu \nu} \bar{c}^{a} \bar{D}_{\mu}^{a b} D_{\nu}^{b c} c^{c}$ in a covariant gauge. Since $\Gamma^{(2)}$ is obtained by differentiation with respect to the dynamical fields, the gauge fixing terms do not generate interaction terms between gravity and the gauge field ghosts.

Hence the key input in the flow equation is the full propagator, $1 /\left(\Gamma_{k}^{(2)}+R_{k}\right)$ for the gauge field $A$ and the metric field $g$. We introduce

$$
\Gamma_{k}^{(2)}=\left(\begin{array}{cc}
\Gamma_{A A}^{(2)} & \Gamma_{A g}^{(2)} \\
\Gamma_{g A}^{(2)} & \Gamma_{g g}^{(2)}
\end{array}\right) \quad \text { and } \quad R_{k}=\left(\begin{array}{cc}
R_{A} & 0 \\
0 & R_{g}
\end{array}\right)
$$

with the convention $\Gamma_{\varphi_{i} \varphi_{j}}^{(2)} \equiv \frac{\delta^{2} \Gamma_{k}}{\delta \varphi_{i} \delta \varphi_{j}}$ for $\varphi=\{A, g\}$. Note that we have chosen a regulator $R_{k}$ which is diagonal. An important observation is that the off-diagonal terms of $\Gamma^{(2)}$ in (19) vanish for $A=\bar{A}=0$ and $g=\bar{g}=\eta$ since the gauge field part in our ansatz involves at least two gauge fields. Consequently, the inverted matrix reads

$$
\left.\frac{1}{\Gamma_{k}^{(2)}+R_{k}}\right|_{\substack{A=\bar{A}=0 \\
g=\bar{g}=\eta}}=\left(\begin{array}{cc}
\frac{1}{\Gamma_{A A}^{(2)}+R_{A A}} & 0 \\
0 & \frac{1}{\Gamma_{g g}^{(2)}+R_{g}}
\end{array}\right) .
$$

We specify the regulator functions and their tensor structures,

$$
\begin{aligned}
R_{A} & =Z_{A} T_{A}(p) p^{2} r_{A}\left(p^{2} / k^{2}\right), \\
R_{g} & =\frac{Z_{N}}{32 \pi G_{N}} \frac{1}{2} T_{g}(p) p^{2} r_{g}\left(p^{2} / k^{2}\right) .
\end{aligned}
$$

Here $r\left(p^{2} / k^{2}\right)$ denotes the shape for the scalar part of the momentum cutoff, which can range between $0 \leq r(y) \leq \infty$ for $\infty \geq y \geq 0$. Both regulators are dressed with the appropriate wave function renormalisation factor to ensure that $R$ displays the same RG scaling as $\Gamma^{(2)}$. The tensor structures $T_{A}$ and $T_{g}$ are chosen as

$$
\begin{aligned}
T_{\mu \nu}^{A}= & \eta_{\mu \nu}-\left(1-\frac{1}{\xi}\right) \frac{p_{\mu} p_{\nu}}{p^{2}} \\
T_{(\alpha \beta)(\gamma \delta)}^{g}= & \frac{\alpha-1}{\alpha}\left[2\left(\eta_{\gamma \delta} \frac{p_{\alpha} p_{\beta}}{p^{2}}+\eta_{\alpha \beta} \frac{p_{\gamma} p_{\delta}}{p^{2}}\right)-\eta_{\beta \gamma} \frac{p_{\alpha} p_{\delta}}{p^{2}}-\eta_{\beta \delta} \frac{p_{\alpha} p_{\gamma}}{p^{2}}-\eta_{\alpha \gamma} \frac{p_{\beta} p_{\delta}}{p^{2}}-\eta_{\alpha \delta} \frac{p_{\beta} p_{\gamma}}{p^{2}}\right] \\
& +\left[\eta_{\alpha \gamma} \eta_{\beta \delta}+\eta_{\alpha \delta} \eta_{\beta \gamma}+\frac{1-2 \alpha}{\alpha} \eta_{\alpha \beta} \eta_{\gamma \delta}\right]
\end{aligned}
$$


with $T_{(\alpha \beta)(\gamma \delta)}^{g}=T_{(\gamma \delta)(\alpha \beta)}^{g}$, and $T_{A}$ diagonal in colour space. We extract the graviton contributions to the running for the YM coupling from the graviton contributions to the running of the YM propagator. The relevant diagrams on the rhs of the flow equation are

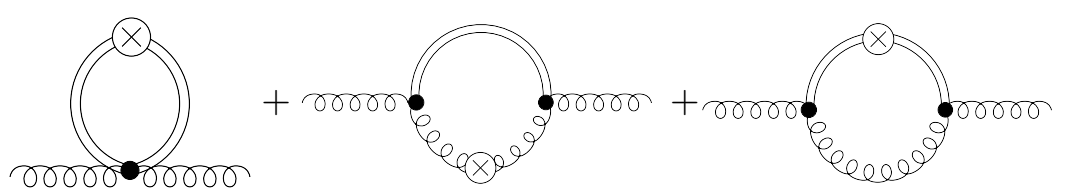

where double lines denote the full graviton propagator, curly lines the full gluon propagator, full dots the full vertices, and the crossed circle the appropriate regulator insertion $\partial_{t} R_{k}$.

Now we come to an important observation. There is a kinematical identity which links the tadpole diagram, the first term in (25), to the other two self energy terms in (25). This identity is expressed diagramatically in (26): It states that the contributions to the tree level gluon-gluon-graviton-graviton amplitude from the 4-point vertex and from the 3-point vertices are proportional to each other when averaged over their angular dependencies. This holds for arbitrary gauge fixing parameter $\alpha$, and in the presence of a cosmological constant. This result can be written as

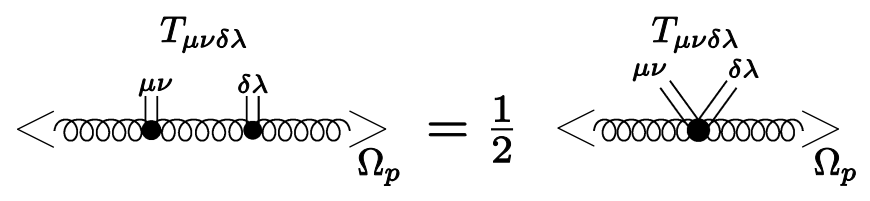

and holds for contractions of the open-ended graviton legs with any of the tensor structures $T_{\mu \nu \delta \lambda}$ of the graviton propagator itself. The brackets indicate that this equality is only valid after the integration over $\int d \Omega_{p}$.

The relation (26) relates to the fact that the graviton-gluon vertices are derived from the classical kinetic term of Yang-Mills theory. This approximation to the effective action of Yang-Mills certainly holds at the Gaußian, asymptotically free fixed point of Yang-Mills. We conclude that it should also hold in the presence of gravity if the gravity contributions to Yang-Mills sustain asymptotic freedom. We shall use this selfconsistency argument later.

A general regularisation scheme may break explicitly or implicitly the relation (26) as it modifies either the propagation or the dynamics of the theory, or both, by cut-off effects. For example, a regularisation scheme in momentum space only changes the propagator, whereas a cutoff in covariant momenta changes propagators and vertices, and might require even treelevel counter terms. In turn, a symmetry preserving regularisation maintains the symmetry relation (26) at the quantum level.

In order to extract the flow equation for the gluon wavefunction renormalisation $\partial_{t} Z_{A}$ we project both sides of the flow equation onto the transversal part of the inverse gluon propagator with $\Pi_{\mu \nu}^{T}=\left(\eta_{\mu \nu}-\frac{q_{\mu} q_{\nu}}{q^{2}}\right)$. Equating the coefficients in front of $q^{2}$ we identify the gravitational contribution to the flow for the gluon wave function renormalisation as

$$
\frac{\partial_{t} Z_{A}}{Z_{A}}=48 \pi^{3} \frac{G_{N}}{Z_{N}} \int_{0}^{\infty} \frac{d p p}{(2 \pi)^{4}} \frac{(1+\alpha)}{\left(1+r_{g}\right)}\left[\frac{\partial_{t}\left(Z_{A} r_{A}\right)}{Z_{A}\left(1+r_{A}\right)^{2}}+\frac{\partial_{t}\left(Z_{N} r_{g}\right)}{Z_{N}\left(1+r_{A}\right)\left(1+r_{g}\right)}-\frac{\partial_{t}\left(Z_{N} r_{g}\right)}{Z_{N}\left(1+r_{g}\right)}\right] .
$$

In (27) we have dropped terms proportional to the flow of the gravitiational gauge fixing parameter, $\partial_{t} \alpha$, its impact will be discussed later. Note also that there are no terms proportional 
to $\xi$ and $\partial_{t} \xi$ due to transversal projections related to the tensor structure of the graviton -gluon vertices. With the anomalous dimensions $\eta_{A}$ and $\eta_{N}$, see (6) and (7) respectively, we deduce from (27) that

$$
\eta_{A, \text { grav }}=-\frac{3 I}{\pi} g_{N} \quad \text { with } \quad I=I_{0}-\frac{\eta_{A}}{2} I_{1} .
$$

with the dimensionless running Newton coupling defined in (5). The coefficients

$$
\begin{aligned}
& I_{0}=\int_{0}^{\infty} d x \frac{1+\alpha}{1+r_{g}} \frac{r_{A}}{1+r_{A}}\left(1+\frac{1}{2} \frac{\eta_{N} r_{g}}{1+r_{g}}\right) \\
& I_{1}=\int_{0}^{\infty} d x \frac{1+\alpha}{1+r_{g}} \frac{r_{A}}{\left(1+r_{A}\right)^{2}}
\end{aligned}
$$

originate from the radial momentum integration with $x=p^{2} / k^{2}$. As discussed before, they only depend on the gravitational gauge fixing parameter but not on the Yang-Mills gauge fixing parameter. The dropped term proportional to $\partial_{t} \alpha$ would effectively lower the coefficient $1 / 2$ in front of the $\eta_{N}$-term in $I_{0}$. To see this we remark that in leading order the gauge fixing term is $k$-independent. This entails that the gauge fixing parameter runs like the wave function renormalisation, $\partial_{t} \ln \alpha \propto-\eta_{N}$. As the prefactor of the $\eta_{N}$-term comes from a summation over all tensor component, the running of $\alpha$ effectively removes the gauge fixing direction from this sum and hence lowers the prefactor. This structure is also present beyond leading order, indeed, for $\alpha=0$ the related term even vanishes identically. Thus, for the sake of simplicity we shall drop this term as it does not change the arguments presented here. Solving (28) for $\eta_{A \text {,grav }}$ we arrive at

$$
\eta_{A}=\eta_{A, \mathrm{YM}}+\eta_{A, \mathrm{grav}}=\frac{\eta_{A, \mathrm{YM}}-\frac{3}{\pi} g_{N} I_{0}}{1-\frac{3}{2 \pi} g_{N} I_{1}}
$$

We conclude from (31) with the coefficients (29), (30) that the gravity-induced running of Yang-Mills is not universal. Note however that the regulators in (29) and (30) in general, do not respect the symmetry constraint (26).

\section{PERTURBATION THEORY}

The one-loop perturbative results are recovered from the flow equation by using the bare two-point functions on the right-hand side of the flow. This corresponds to setting $Z_{A}=$ $Z_{N}=1$ on the right hand side of (31). Then the one-loop graviton correction to the gluon anomalous dimension reads

$$
\eta_{A, \text { grav }}=-\frac{3 g_{N}}{\pi} I_{0,1-\mathrm{loop}}
$$

with

$$
I_{0,1-\text { loop }}=\int_{0}^{\infty} d x \frac{1+\alpha}{1+r_{g}(x)} \frac{r_{A}(x)}{1+r_{A}(x)} .
$$

$I_{0,1-\text { loop }}$ encodes the gauge-fixing and regularisation dependence of the one-loop coefficient. It is crucial to observe that

$$
\left.\eta_{A, \text { grav }}\right|_{1-\text { loop }} \leq 0
$$


for $\alpha \geq-1$, and all regulators. We conclude that the full one-loop running of Yang-Mills is not universal due to the gravity correction. However, it sustains asymptotic freedom for all regularisations. Furthermore it has both, a regulator as well as a gravity gauge dependence via $\alpha$. Both are not independent as the latter one can be absorved within a specific choice of the regulator. This is not surprising as the regulator can be also partially viewed as a change of the gauge fixing. In order to disentangle these effects we first consider a vanishing gravity regularisation, $r_{g} \equiv 0$. The gauge dependence persists, $\partial_{\alpha} \eta_{A, \text { grav }}=\eta_{A \text {,grav }} /(1+\alpha)$ at fixed Newton coupling. Hence, $\eta_{A}$ is only independent of $\alpha$ for $\eta_{A \text {,grav }} \equiv 0$, which is equivalent to the constraint

$$
\frac{1}{1+r_{g}} \frac{r_{A}}{1+r_{A}} \equiv 0
$$

The constraint (35) implies that $1 / r_{g}$ has to vanish for finite $r_{A}>0$. Consequently, $r_{g}$ is a sharp cutoff for momenta where $r_{A}>0$. Legitimate choices are for example $r_{g}=r_{A}=r_{\text {sharp }}$, or $r_{g}=r_{\text {sharp }}$ and $r_{A}=r_{\text {opt }}$ [32]. The sharp and optimised regulators are defined as

$$
\begin{aligned}
r_{\text {sharp }}(x) & =\frac{1}{\theta(x)}-1, \\
r_{\text {opt }}(x) & =\left(\frac{1}{x}-1\right) \theta(1-x),
\end{aligned}
$$

and the Heaviside step function $\theta$. Note that regulators with the constraint (35) effectively satisfy the symmetry constraint (26): if the graviton legs in (26) are contracted with graviton propagators, we are lead to (35). In turn, without graviton propagators the constraint (26) necessitates $r_{A}=0$.

We summarise the above analysis as follows: we have computed the one loop gravity contributions $\eta_{A \text {,grav }}$ to the Yang-Mills $\beta$-function for general regularisation schemes. The one-loop gravitational contribution to the running of the YM coupling is regularisationdependent, and thus non-universal. This originates from the mass dimension inherent to the gravitational coupling. However, $\beta_{\mathrm{YM}, \text { grav }}$ is negative semi-definite and hence sustains asymptotic freedom. It vanishes in symmetry-preserving regularisation schemes based on the symmetry relation (26).

\section{ASYMPTOTIC FREEDOM AND QUANTUM GRAVITY}

In this section we extend the stability analysis of asymptotic freedom to include general field-theory-based gravity scenarios $33-44,46$ 50]. In some of these, short distance physics is shielded by black hole formation [47 50]. Provided this happens at scales where the anomalous dimension of the graviton $\eta_{N}$ is still small, the weak coupling analysis of the previous section is sufficient to sustain asymptotic freedom of Yang Mills. However, since the graviton anomalous dimension $\eta_{N}$ might grow large due to strong quantum gravity effects, it is important to evaluate the stability of asymptotic freedom for general $\eta_{N}$.

From the RG-equation for $g_{N}$, (17), we note that the value $\eta_{N}=-2$ is distinguished because the $\beta$-function for the dimensionless Newton coupling $g_{N}$ vanishes. This is the gravitational fixed point of the asymptotic safety scenario for gravity [33 44, 46]. In its vicinity, the 
gravitational $\beta$-function (77) changes sign. Therefore the three cases $\eta_{N}=-2, \eta_{N}<-2$ and $\eta_{N}>-2$ are qualitatively different and will be discussed separately.

We first discuss the impact of a gravitational fixed point $\eta_{N}=-2$ for asymptotic freedom of Yang-Mills by evaluating the gravitational contribution $\eta_{A \text {,grav }}$, (28). The related coefficient $I_{0}$ in (29) becomes

$$
I_{0}=\int_{0}^{\infty} d x \frac{1+\alpha}{\left(1+r_{g}\right)^{2}} \frac{r_{A}}{\left(1+r_{A}\right)}
$$

and the coefficient $I_{1}$ is given by (30). With the coefficients $I_{0}$ and $I_{1}$ and the assumption of asymptotic freedom, $g_{\mathrm{YM}} \equiv 0$ at the fixed point, the $\beta$-function at the fixed point (31) reads

$$
\eta_{A *}=-\frac{\frac{3}{\pi} g_{N_{*}} I_{0}}{1-\frac{3}{2 \pi} g_{N_{*}} I_{1}}
$$

As for the one loop running, $\eta_{A_{*}}$ is not universal since the coefficients $I_{0}, I_{1}$ depend on the choices for the regulators $r_{g}, r_{A}$. Asymptotic freedom only enforces $\eta_{A_{*}} \leq 0$ which implies

$$
I_{0} \geq 0 \quad \wedge \quad g_{N_{*}} \leq \frac{2 \pi}{3 I_{1}}, \quad \text { or } \quad I_{0}<0 \quad \wedge \quad g_{N_{*}}>\frac{2 \pi}{3 I_{1}}
$$

The latter case is irrelevant here as $I_{0}<0$ can only be obtained for the singular choices $\alpha<-1$. For the symmetry preserving choices (35) we have $I_{0}=0$ and we arrive at a vanishing non-perturbative gravity contribution for the Yang-Mills $\beta$-function, $\eta_{A_{*}}=0$.

In the general case we have to satisfy the constraint in (40) relating $I_{1}$ and $g_{N_{*}}$. For the sake of simplicity we restrict ourselves to regulators with $r_{A}(x \geq 1) \equiv 0$ as this limits the size of the back-reaction of the gluon on the graviton. Then, $I_{1}$ in (30) satisfies

$$
I_{1}=\int_{0}^{1} d x \frac{1+\alpha}{1+r_{g}} \frac{r_{A}}{\left(1+r_{A}\right)^{2}} \leq \frac{1}{4} \frac{1+\alpha}{1+r_{g, \min }}
$$

where $r_{g, \min }=\min r_{g}(x)$ with $x \in[0,1]$. We have also used that $r_{A} /\left(1+r_{A}\right)^{2} \leq 1 / 4$. Due to the bound (41) the first set of constraints in (40) is satisfied for

$$
g_{N *} \leq \frac{8 \pi}{3} \frac{1+\alpha}{1+r_{g, \min }} .
$$

The bound (42) for $g_{N_{*}}$ is safely satisfied for general regulators $r_{g}$. Indeed, the generic value for the fixed point coupling is of order one. For example, for the pure gravity system one finds $g_{N_{*}}=0.893$ for an optimised regulator and $\alpha=0$. Note also in this context that $g_{N_{*}}(1+\alpha)$ is approximately constant for all $\alpha$. If we relax the locality condition for the gluon, that is $r_{A}(x \geq 1) \not \equiv 0$, one enhances the gluon fluctuations. In this case one has to take into account the back-reaction of the gluon fluctuations on the fixed point value of the Newton coupling. Here we only remark that in the full system indeed one can show that $g_{N_{*}} \leq \frac{2 \pi}{3 I_{1}}$ is always satisfied. This will be discussed in more detail elsewhere.

In summary we find that the graviton contribution $\eta_{A \text {,grav }}$ sustains asymptotic freedom

$$
\eta_{A, \operatorname{grav}_{*}} \leq 0
$$


Hence the ultraviolet fixed point action of Yang-Mills theory is given by the classical action and we can invoke (26) leading to $\eta_{A \text {,grav }}=0$. This leads us to the final value of the nonperturbative $\beta$-function if all symmetries are respected,

$$
\eta_{A *}=0
$$

We conclude that the gauge coupling remains asymptotically free, even in the presence of a non-perturbative gravitational fixed point.

Next we turn to $\eta_{N}<-2$. In this case $I_{0}$ might turn negative, see (29). However, with (17) we have $\beta_{g_{N}}<0$ and $g_{N}$ decreases exponentially with logarithmic RG scale $t=\ln k$, and so does the gravity contribution to $\eta_{A}$ in (31). We conclude that even though the gravity contribution to $\eta_{A}$ might be positive for $\eta_{N}<-2$, its exponential decay would eventually lead to the dominance of $\eta_{A, Y M}$. Thus, asymptotic freedom would persist. Note also that $\eta_{N}<-2$ for $t \rightarrow \infty$ is equivalent with a Gaußian UV fixed point which is not present in gravity.

Finally, for $\eta_{N}>-2$ the dimensionless Newton coupling $g_{N}$ grows exponentially with $t$, see (17), and the coefficient $I_{0}$ is positive. This case includes classical gravity with $\eta_{N}=0$ as well as a gravitational shielding scenario. The constraints (40) enforce $g_{N} \leq 2 \pi /\left(3 I_{1}\right)$ to ensure asymptotic freedom for Yang-Mills. This puts an upper limit on the value of dimensionless Newton coupling which has to be satisfied by eg. perturbation theory or a gravitational shielding scenario, or else $g_{N}$ de-stabilizes asymptotic freedom. Note that this bound depends on the regularisation scheme as does the definition of the dimensionless Newton constant $g_{N}$.

In summary we have shown that asymptotic freedom persists in general field-theory-based gravity scenarios.

\section{COSMOLOGICAL CONSTANT}

We proceed by introducing a cosmological constant $\Lambda$ to the theory. For the sake of simplicity we consider the case $\alpha=0$, but the results readily extend to $\alpha \neq 0$. The coefficient functions $I_{0}$ and $I_{1},(28)$, read

$$
\begin{aligned}
& I_{0}=\int_{0}^{\infty} d x \frac{1}{1-\frac{2 \lambda}{x}+r_{g}} \frac{r_{A}}{1+r_{A}}\left(1+\left(\frac{\eta_{N} r_{g}}{2}-\frac{2 \lambda+\partial_{t} \lambda}{x}\right) \frac{1}{1-\frac{2 \lambda}{x}+r_{g}}\right) \\
& I_{1}=\int_{0}^{\infty} d x \frac{1}{1-\frac{2 \lambda}{x}+r_{g}} \frac{r_{A}}{\left(1+r_{A}\right)^{2}}
\end{aligned}
$$

where $\lambda=\Lambda_{k} / k^{2}$ denotes the cosmological constant in units of the RG scale $k$. This leads to the beta-function (39) with the coefficients $I_{0}$ and $I_{1}$ in (45). As before we conclude that if invoking the symmetry relation (26) leading to (35) with $1+r_{g} \rightarrow 1-2 \lambda / x+r_{g}$, the coefficient function (45) vanishes, $I \equiv 0$, and we have no gravity contribution to the Yang-Mills running,

$$
\eta_{A, \mathrm{grav}}=0
$$

It is left to prove the stability of this result under changes of the regulator, that is $\eta_{A, \text { grav }} \leq 0$ for all regulators. 
As in the previous section we consider the qualitatively different cases $\eta_{N}=-2, \eta_{N}<-2$ and $\eta_{N}>-2$. At the gravitational fixed point with $\eta_{N}=-2$ and $\partial_{t} \lambda=0$ we find

$$
I_{0}=\int_{0}^{\infty} d x \frac{r_{A}}{1+r_{A}} \frac{1}{\left(1-\frac{2 \lambda}{x}+r_{g}\right)^{2}}\left(1-\frac{4 \lambda}{x}\right) .
$$

We have stability of the fixed point iff $I_{0} \geq 0$. Evidently the integrand in (47) is not positive but is negative for small momenta $x$ and turns positive for $x>4 \lambda$. For regulators (36), (37) we find stability for $\lambda \leq \lambda_{\text {crit }}=\frac{1}{8}$. For general regulators one has to solve the integral equation $I_{0}$ numerically resulting in $\lambda_{\text {crit }}\left(r_{g}, r_{A}\right)$ and the constraint $\lambda \leq \lambda_{\text {crit }}\left(r_{g}, r_{A}\right)$. The regulator-dependence of the critical cosmological constant is not surprising as the value of the cosmological constant (and the Newton constant) at the fixed point is not a physical observable. The important result is the existence of such a constraint.

For $\eta_{N}<-2$ and $\eta_{N}>-2$ we run into the same scenarios as in the previous section with the coefficients $I_{0}$ and $I_{1}$. Note, however, that for $\eta_{N}>-2$ we require $I_{0}>0$ which puts constraints on $\lambda$ and $\partial_{t} \lambda$. As one can assume $\partial_{t} \lambda<0$ due to the canonical running in this regime, they are less severe than the one in the asymptotic safety scenario.

\section{DISCUSSION}

First we compare our results with previous findings to leading order in perturbation theory in the $U(1)$ and $S U(N)$ cases $[3-6,8,10,11,16]$. Identifying the energy scale $E$ with the cutoff scale $k$, the one-loop gravitational correction can be written as

$$
\beta_{\mathrm{YM}, \text { grav }}=-\frac{3 I}{2 \pi} g_{\mathrm{YM}} G_{N} E^{2} .
$$

Our result corresponds to the coefficient $I=I_{1-\text { loop }}$ as given in (33). Within dimensional regularisation and using the background field method, Deser et. al. [10, 11] found no gravitational corrections to the Yang-Mills and Maxwell $\beta$-functions, respectively, and hence $I=0$. In the $U(1)$ case, the same conclusion is reached by Pietrykowski [4] by evaluating the gauge fixing dependence in a generalised $R_{\xi}$ gauge, and by Toms [5] within the geometrical effective action method. In the $S U(N)$ case, Ebert, Plefka and Rodigast [6] confirmed $I=0$ based on dimensional regularisation and cutoff regularisation with Feynman gauge for the gluon and de Donder gauge for the graviton. This study shows that quadratic divergences, neglected within dimensional regularisation, cancel out within a sharp cutoff regularisation.

On the other hand, Robinson and Wilzcek [3], Toms [8] and Daum et. al. [16] find a non-vanishing contribution to leading order, $I>0$. In particular, the result of Robinson and Wilczek [3] is based on the Feynman gauge $\xi=1$ and $\alpha=1$, leading to $I_{\mathrm{RW}}=2$ in (48). A positive coefficient $I_{\mathrm{T}}=\frac{2}{3}$ has also been found by Toms [8] using a modified version of the Vilkovisky de-Witt approach. The study of Daum, Harst and Reuter [16] is based on the background field method, together with a Wilsonian momentum cutoff for the propagating modes, with

$$
I_{\mathrm{DHR}}=4 \int_{0}^{\infty} d x \frac{-x^{2} r^{\prime}(x)}{1+r(x)}=8 \int_{0}^{\infty} d x x \ln [1+r(x)]
$$


Here $r(x)$ parametrises the shape of the momentum cutoff. The coefficient is non-universal and strictly positive, $I_{\mathrm{DHR}}>0$, as $r^{\prime} \not \equiv 0$. As has been argued in Sec. IV, the background field approach used in this study does also include terms originating in the background field dependence of the regulator. For the symmetry-preserving cut-off choice $r_{\text {sym }}$ with (35) the coefficient (49) diverges in clear contradistinction to our result $I\left[r_{\text {sym }}\right]=0$. This difference can be solely attributed to the field-dependence of the cut-off function which enters (49).

In the presence of a cosmological constant, a recent study based on a diffeomorphism invariant expansion scheme using the geometric effective action finds a non-vanishing result to one-loop order [15]. The expansion scheme used in this approach differs from the standard one as eg. already the Yang Mills classical propagator is massive with a mass proportional to the cosmological constant. We emphasize that the symmetry preserving scheme employed here also leads to a vanishing coefficient in this set-up, $I=0$.

Based on the general considerations underlying our RG result, we can acertain that the gauge-fixing and regularisation-dependent coefficient $I_{1-\text { loop }} \geq 0$. As long as the implicit or explicit regularisation respects the symmetry relation (26) , the result reads $I=0$. Note in this context that regularisations such as Pauli-Villars, or other gauges such as a general $R_{\xi}$ gauge, do not respect the symmetry constraint. We also remark that in the computation of a nonuniversal coefficient one cannot tell apart regularisation and gauge-fixing dependences. It is therefore not surprising that different regularisations and gauges lead to different coefficients $I \geq 0$. We conclude that all existing studies agree in that the gravitational contribution to the Yang-Mills $\beta$-function support asymptotic freedom.

The one-loop approximation is valid in the weak gravity regime. Close to the Planck scale and beyond, the dynamics within the gravitational sector becomes relevant. We have shown that asymptotic freedom persists for general anomalous dimension $\eta_{N}$, which entails its compatibility with general field-theory-based gravity scenarios. This includes gravitational shielding as well as asymptotically safe gravity.

In conclusion, provided that all symmetries and in particular (26) are preserved, the graviton induced running of the Yang-Mills coupling vanishes,

$$
\left.\eta_{A}\right|_{\text {grav }}=0
$$

This result stays valid in the presence of a cosmological constant, and in the presence of a gravitational fixed point. Hence, it will be interesting to extend this study to the fully coupled Yang-Mills gravity system.

\section{Acknowledgments}

This work is supported by Helmholtz Alliance HA216/ EMMI, and by the Science and Technology Research Council [grant number ST/G000573/1].

[1] D. J. Gross, F. Wilczek, Phys. Rev. Lett. 30 (1973) 1343-1346.

[2] H. D. Politzer, Phys. Rev. Lett. 30 (1973) 1346-1349. 
[3] S. P. Robinson and F. Wilczek, Phys. Rev. Lett. 96, 231601 (2006) arXiv:hep-th/0509050].

[4] A. R. Pietrykowski, Phys. Rev. Lett. 98, 061801 (2007) arXiv:hep-th/0606208.

[5] D. J. Toms, Phys. Rev. D 76, 045015 (2007) arXiv:0708.2990 [hep-th]].

[6] D. Ebert, J. Plefka and A. Rodigast, Phys. Lett. B 660, 579 (2008) arXiv:0710.1002 [hep-th]].

[7] Y. Tang and Y. L. Wu, arXiv:0807.0331 [hep-ph].

[8] D. J. Toms, Nature 468 (2010) 56-59. arXiv:1010.0793 [hep-th]].

[9] J. F. Donoghue, Phys. Rev. Lett. 72 (1994) 2996 gr-qc/9310024].

[10] S. Deser, H. -S. Tsao, P. van Nieuwenhuizen, Phys. Lett. B50 (1974) 491.

[11] S. Deser, P. van Nieuwenhuizen, Phys. Rev. D10 (1974) 401.

[12] S. Deser, H. -S. Tsao, P. van Nieuwenhuizen, Phys. Rev. D10 (1974) 3337.

[13] D. Ebert, J. Plefka and A. Rodigast, arXiv:0809.0624 [hep-ph].

[14] F. Wu and M. Zhong, arXiv:0809.1913 [hep-ph].

[15] D. J. Toms, Phys. Rev. Lett. 101, 131301 (2008) arXiv:0809.3897 [hep-th]].

[16] J. -E. Daum, U. Harst, M. Reuter, JHEP 1001 (2010) 084. arXiv:0910.4938 [hep-th]].

[17] J. Ellis, N. E. Mavromatos, arXiv:1012.4353 [hep-th]].

[18] C. Wetterich, Phys. Lett. B 301 (1993) 90; J. Berges, N. Tetradis and C. Wetterich, Phys. Rept. 363 (2002) 223 hep-ph/0005122.

[19] D. F. Litim and J. M. Pawlowski, in: The Exact Renormalization Group, Eds. Krasnitz et al, World Sci (1999) 168 hep-th/9901063.

[20] H. Gies, arXiv:hep-ph/0611146.

[21] J. M. Pawlowski, Annals Phys. 322 (2007) 2831 arXiv:hep-th/0512261.

[22] D. F. Litim and J. M. Pawlowski, JHEP 0209, 049 (2002) [arXiv:hep-th/0203005].

[23] H. Gies, Phys. Rev. D 66 (2002) 025006 arXiv:hep-th/0202207.

[24] J. M. Pawlowski, D. F. Litim, S. Nedelko and L. von Smekal, Phys. Rev. Lett. 93 (2004) 152002 hep-th/0312324;

[25] M. Reuter and C. Wetterich, Phys. Rev. D 56 (1997) 7893 hep-th/9708051;

[26] D. F. Litim and J. M. Pawlowski, Phys. Lett. B 435 (1998) 181.

[27] F. Freire, D. F. Litim and J. M. Pawlowski, Phys. Lett. B 495, 256 (2000);

[28] J. M. Pawlowski, Int. J. Mod. Phys. A 16 (2001) 2105.

[29] J. M. Pawlowski, arXiv:hep-th/0310018.

[30] D. F. Litim and J. M. Pawlowski, Phys. Rev. D 66, 025030 (2002) arXiv:hep-th/0202188.

[31] D. F. Litim and J. M. Pawlowski, Phys. Lett. B 546, 279 (2002) arXiv:hep-th/0208216.

[32] D. F. Litim, Phys. Lett. B486 (2000) 92-99. hep-th/0005245. Phys. Rev. D64 (2001) 105007. hep-th/0103195.

[33] S. Weinberg, in General Relativity: An Einstein centenary survey, Eds. S.W. Hawking and W. Israel, Cambridge University Press (1979), p. 790; arXiv:0903.0568 [hep-th]; arXiv:0908.1964 [hepth]; arXiv:0911.3165 [hep-th].

[34] M. Niedermaier, Class. Quant. Grav. 24 (2007) R171 gr-qc/0610018; M. Niedermaier and M. Reuter, Living Rev. Relativity 9 (2006) 5.

[35] R. Percacci, in 'Approaches to Quantum Gravity: Towards a New Understanding of Space, Time and Matter' ed. D. Oriti, Cambridge University Press, 0709.3851 [hep-th].

[36] D. F. Litim, PoS QG-Ph (2008) 024, 0810.3675 [hep-th].

[37] M. Reuter, Phys. Rev. D 57 (1998) 971 hep-th/9605030].

[38] W. Souma, Prog. Theor. Phys. 102 (1999) 181 [hep-th/9907027].

[39] O. Lauscher and M. Reuter, Phys. Rev. D 65 (2002) 025013 [hep-th/0108040]; Class. Quant. Grav. 19 (2002) 483 hep-th/0110021; Phys. Rev. D 66 (2002) 025026 hep-th/0205062.

[40] R. Percacci and D. Perini, Phys. Rev. D 67 (2003) 081503 hep-th/0207033]; Phys. Rev. D 68 (2003) 044018 hep-th/0304222];

[41] D. F. Litim, Phys. Rev. Lett. 92 (2004) 201301 hep-th/0312114]. AIP Conf. Proc. 841 (2006) 322 arXiv:hep-th/0606044.

[42] P. Fischer and D. F. Litim, Phys. Lett. B 638 (2006) 497 arXiv:hep-th/0602203; AIP Conf. Proc. 861 (2006) 336 arXiv:hep-th/0606135. 
[43] A. Codello, R. Percacci and C. Rahmede, Int. J. Mod. Phys. A 23 (2008) 143 arXiv:0705.1769 [hep-th]]; Annals Phys. 324 (2009) 414 [arXiv:0805.2909 [hep-th]].

[44] D. Benedetti, P. F. Machado and F. Saueressig, Nucl. Phys. B 824 (2010) 168 arXiv:0902.4630 [hep-th]].

[45] S. Folkerts, Diploma thesis, University of Heidelberg (August 2009).

[46] M. R. Niedermaier, Phys. Rev. Lett. 103 (2009) 101303; PoS CLAQG (2008) 05.

[47] T. Banks and W. Fischler, arXiv:hep-th/9906038.

[48] S. B. Giddings, S. D. Thomas, Phys. Rev. D65 (2002) 056010. hep-ph/0106219.

[49] G. Dvali and C. Gomez, arXiv:1005.3497 [hep-th].

[50] G. Dvali, S. Folkerts and C. Germani, Phys. Rev. D 84 (2011) 024039 arXiv:1006.0984 [hep-th]].

[51] R. Percacci and G. P. Vacca, arXiv:1008.3621 [hep-th]. 